\title{
AGENT BASED MODELING: FINE-SCALE SPATIO-TEMPORAL ANALYSIS OF PERTUSSIS
}

\author{
D. A. Mills* \\ Department of Geography, Texas State University, 601 University Dr. San Marcos, TX 78666 - dam203@txstate.edu
}

KEY WORDS: Agent Based Modeling, Spatio-Temporal Analysis, open-source, Pertussis, infectious diseases, vaccination effectiveness

\begin{abstract}
:
In epidemiology, spatial and temporal variables are used to compute vaccination efficacy and effectiveness. The chosen resolution and scale of a spatial or spatio-temporal analysis will affect the results. When calculating vaccination efficacy, for example, a simple environment that offers various ideal outcomes is often modeled using coarse scale data aggregated on an annual basis. In contrast to the inadequacy of this aggregated method, this research uses agent based modeling of fine-scale neighborhood data centered around the interactions of infants in daycare and their families to demonstrate an accurate reflection of vaccination capabilities. Despite being able to prevent major symptoms, recent studies suggest that acellular Pertussis does not prevent the colonization and transmission of Bordetella Pertussis bacteria. After vaccination, a treated individual becomes a potential asymptomatic carrier of the Pertussis bacteria, rather than an immune individual. Agent based modeling enables the measurable depiction of asymptomatic carriers that are otherwise unaccounted for when calculating vaccination efficacy and effectiveness. Using empirical data from a Florida Pertussis outbreak case study, the results of this model demonstrate that asymptomatic carriers bias the calculated vaccination efficacy and reveal a need for reconsidering current methods that are widely used for calculating vaccination efficacy and effectiveness.
\end{abstract}

\section{INTRODUCTION}

Recently, scientifically undisputed research has shown that it is probable that the acellular Pertussis vaccination fails to prevent colonization and transmission of pertussis bacteria in humans (Warfel, 2013). "Asymptomatic transmission is the most parsimonious explanation for many of the observations surrounding the resurgence of B. pertussis in the US and UK" (Althouse, 2015). Although agent based models have simulated Pertussis and other infectious diseases, none have incorporated or evaluated the potential risk of asymptomatic carriers as defined by Warfel's recent research. "With the relatively new field of agent-based modeling, factors that are difficult to measure directly (undetected infections, adult immunity, and waning immunity) can be investigated with computer simulations that can capture high levels of detail" (Sanstead 2015). This study's primary research objective is to reconcile the diversity of endogenous and exogenous factors of a pertussis outbreak case study through the iterative and comparative processing of a uniquely developed, fine-scale, and detailoriented agent based model.

Contemporary debates concerning infectious disease and vaccination are typically examined as a phenomenon that assumes higher vaccination rates equal lower infectious disease prevalence. Often, these phenomena are evaluated using differential equations and the direct application of regression models to coarse aggregated data. In contrast to these methods, agent based modeling can reveal complex emergent properties which are beyond the scope of results derived using differential equations and regression modeling alone. This research utilizes open-source Netlogo 6.0 software that is developed and distributed by Northwestern University. Netlogo is a modern

\footnotetext{
*Corresponding author
}

programming language that is very well suited for developing agent based models of infectious disease transmission (Just 2015). Using Netlogo and empirical data from a Leon County, Florida daycare case study, this research follows fundamental tenets of traditional and contemporary mathematical modeling theory to identify the large-scale impact of asymptomatic carriers caused by acellular Pertussis vaccination.

Agent based modeling is a relatively new field and its application to epidemiological research has yet to be fully realized. This freely available open-source model offers researchers and medical professionals a new tool and theoretical method for measuring confounding variable impact on Pertussis disease spread. In a broad scope, this research extends the understanding of how agent based modeling can benefit researchers in the analysis of epidemiological issues, such as acellular Pertussis vaccination efficacy and effectiveness.

\section{BACKGROUND}

Sir Francis Galton is documented as having presented the first ever linear regression line in a lecture given in 1877 (Pearson, 1930). In epidemiology, some of the most common regression models are simple linear regression, multiple linear regression, generalized linear models, and multivariate generalized linear models. In epidemiology, simple linear regression can be used to measure trends in disease indicators (Guimarães 2012). Results of this model apply plotting and regression analysis to create effective visualization and descriptive data from model outcomes.

In 1944 John Von Neumann and O. Morgenstern were reviewed as having begun a "radically new direction in constructing a mathematical analysis of Human Behavior" (E, 1945). After Von 
Neumann's passing in 1957, Morgenstern offered the following summary of the Theory of Games as a game of strategy. Morgenstern wrote, "in a game of strategy, as distinguished from mere games of chance, the outcome is never dependent on the variables controlled by any one individual alone but only by all variables, some of which are controlled by other individuals, some perhaps by chance" (Morgenstern, 1958). Purposefully designed agent based models operate on the fundamental tenets of Von Neumann and Morgenstern's Theory of Games. For the purpose of this research using an agent based model, agents are given characteristics and sets of rules whereby their behaviors and interactions with other agents are shaped dependent on each agent's unique variable combinations, preferences, as well as the attributes of the environment.

Another renowned contributor to mathematical modeling is Thomas C. Schelling. In Game Theory and the Study of Ethical Systems, Schelling wrote, "one can ask whether game theory supposes a certain ethic, or has some stated or unstated ethical assumptions or implications" (Schelling, 1968). Stated or unstated ethical assumptions are an important consideration in agent based modeling. The rules and environments that agents adhere to in an agent based model are programmed by human developers. The potential for ethical bias stemming from the programming of an agent based model is ubiquitous. Deliberate or unconscious bias in an agent based model's development may be prevented through adherence to a framework that insists on empirically evidenced agent rules and transparency of ambiguities and limitations in the documentation of research and programming of the model. The agent based model developed for this research leverages an open-source approach and is freely available for use to ensure transparency, replicability, validation and to maximize the potential for future innovation and improvements of the application for the betterment of medical research. Ultimately, this model intends to serve as a building block toward improved outcomes for those vulnerable populations which have been and continue to be afflicted by Pertussis at an increasing rate.

Many research questions would not benefit from an iterative agent based model approach and instead could be resolved using differential equation modeling. Assumptions regarding vaccination effectiveness and efficacy can create the impression that effective results can be achieved through these aggregated methods. Axelrod imparts a sense of caution regarding this impressionistic thought process by stating, "although the assumptions may be simple, the consequences may not be at all obvious" (Axelrod, 2001). Axelrod then identified a unique outcome derived through agent based modeling. Axelrod wrote, "the large-scale effects of locally interacting agents are called 'emergent properties' of the system" (Axelrod, 2001). This research focuses on the observation and analysis of these locallyinteracting agents to reveal the nature of the large-scale effects of asymptomatic carriers caused by the use of the acellular Pertussis vaccination.

Using agent based modeling, modern research has examined "the impact of undetected infections, adult immunity, and waning vaccine-acquired immunity on recent age-related trends in pertussis incidence" (Sanstead, 2015). The research in Sanstead's study used an agent based model developed in Netlogo 5.0.4. The research successfully fit the model using a case study from a Dakota County outbreak to identify factors that may have contributed to outbreaks in Dakota County. Their research success demonstrated that "agent-based modeling is a useful tool for this (field of) research" (Sanstead, 2015). Sanstead's method of applying a case study to fit the model will be applied in this research using a Florida daycare outbreak.

\section{THEORETICAL FRAMEWORK}

This research will use an empirical positivist theoretical framework. The agent based model systematically assesses pertussis bacteria transmission in a fine-scale environment with detailed spatio-temporal relationships and agent characteristics that are scientifically recognized as pertinent to disease transmission. The agent-based model incorporates bacteria, various agent bacteria carriers, progressive agent aging, symptoms, vaccine schedules, disease progression (incubation to recovery), antibiotics, $\%$ of population vaccinated, vaccination strategy, vaccine efficacy, daily varying temperature, age based agent awareness, varying levels of susceptibility, waning immunity, and asymptomatic carriers. Heuristic inferences are disclosed, customizable via the user interface, and used to develop a range of life-emulating environments and interactions based on unique environmental characteristics.

This study utilizes fine-scale schematic domains representing a transportation network, daycare, homes, schools, and office buildings. Many agent-based models have been developed to analyze the spread of infectious diseases from a large-scale such as a city, state, or country. However, none have examined how vulnerable populations may be impacted from a scale as intimate as a daycare. This fine-scale model (12 - 144 agent individuals represented) is exploratory toward the discovery of specific pathways of underlying endemic patterns that a large-scale model $(50,000+$ agent individuals, i.e. a small city) may not be capable of emulating. Fine-scale data being integrated into an agent based model reveals the large-scale emergent properties of local asymptomatic carriers. Another benefit arising from the availability of this open-source model is that it may potentially be used by social workers, healthcare workers, NonGovernmental Agencies, or other interested parties for demonstrating a Pertussis outbreak to wider community audiences or a body of students.

An important question that is raised by the results of this model is: Are vulnerable populations, which are incapable of safely receiving vaccinations, at greater risk when the potential for a naïve cohort of asymptomatic carriers is created by a vaccine? The systematic investigation through fine-scale mathematical modeling can emulate potential outcomes for affected vulnerable populations. This research intends to contribute to the larger body of epidemiological research regarding the transmission of infectious diseases by providing an open-source spatio-temporal agent based model. Having the capability of mathematically determining a measure of acellular pertussis inoculated asymptomatic carriers and their impact on the spread of Pertussis may reveal important ethical considerations regarding current vaccination strategies. These ethical considerations should be addressed by healthcare professionals, researchers, and policy makers.

\section{DATA}

This research will utilize case study research data from the Florida Department of Health. The Pertussis outbreak in the case study recorded cases from June 2013 to January 2014 in Leon County, Florida. Data from this outbreak was gathered from the article, Sustained Transmission of Pertussis in Vaccinated, 1-5- 
Year-Old Children in a Preschool, Florida, USA (Matthias, 2016). The data details the age range of the students enrolled at the daycare, the number of students enrolled in the daycare, the number of infections and age-groups of infections, and the vaccination rates of the students at the daycare. While protecting private information about people, the data includes the ages of the infected and whether they were up to date with their acellular Pertussis vaccination. Data used for calibration of the model includes the vaccination status of the student and infected child's family, dates confirmed and probable cases, and attack rates.

\section{METHODS}

The model focuses on the interaction of five human agents: a caregiver parent (responsible for taking child to daycare), a noncaregiver parent, a K-12 school aged sibling, a child (0-5 years), and two daycare workers per classroom. An auxiliary output window features a clock, calendar and temperature indicator record and display the month, day, year, 24-hour time, the day of the week (Sunday - Saturday) and temperature. This clock is used to track and record interactions and spread of the Pertussis disease in the model. Four primary location schematics are modeled for agents to interact within: schools, houses, daycare classrooms, and workspaces. A transportation network is created to simulate the caregiver parent taking their child to and from daycare while arriving at different times during the morning. Information regarding attack-rates, vaccination rates, vaccination efficacy, and vaccination effectiveness is continuously tracked during each iteration. The model is programmed to allow customizable functionality and variable adjustment through the user-interface which enables researchers with little to no programming experience to utilize this model commensurate with data available from existing sources and emerging research. Users with programming skills can modify the hard-code using open-source Netlogo software. My contact information is also available in the model's built-in, "how it works", documentation to provide constructive feedback regarding simulation outcomes and offer suggestions or comments toward improvement.

Empirical, scientifically derived rules for agents are implemented, recorded and any identified ambiguities or potentially ambiguous characteristics, attributes, or variables within the model are disclosed. Waning immunity is one example of an empirical rule implemented into the model. This empirical rule is derived from Schwartz's research which states, "the odds of pertussis increased by $27 \%$ each year that passed after receipt of an acellular vaccine" (Schwartz, 2016). This variable is customizable via the user-interface and affects an agents individual vaccine effectiveness which is at a maximum of $85 \%$ on the day their last shot was received at a rate of $1 / 365$ or 1/366 per day depending on the presence of a leap year according to the Gregorian calendar.

Interactions between individual agents are tracked to record if an agent was challenged with bacteria from a vaccinated or unvaccinated agent, whether they defended against the bacteria successfully if they are vaccinated, where the attack occurred, and what time and date the attack occurred. Appropriate colorcoded visualizations are used to best demonstrate the progression of the disease across the daycare community and a final screenshot of each iteration is taken which has the potential for analysis for the future. The model is scalable to create an environment with 12 to 144 children in increments of 12 depending on the size of the community the case study. This study uses 120 children to simulate the Florida daycare outbreak case study which had an enrollment of 117 children.
The model is an exploratory model which demonstrates a method for measuring important potential epidemiological properties for which there is currently no other means of measurement. As the empirical daycare information has been incorporated, the model has been run through many thousand iterations with many thousand more to come in the process of calibrating toward becoming a confirmatory model. As additional daycare and disease information is obtained, the model may evolve into becoming a deterministic or predictive model regarding disease progression. Data is extracted and aggregated using Python and then analyzed using R. All scripts have been retained and are freely available for use by interested parties.

\section{MODEL INFRASTRUCTURE}

\subsection{User Interface}

The model window (Figure 1) is a rectangle, 120 pixels wide by 100 pixels tall. Coordinates range from $(0,50)$ to $(120,-50)$. Each patch measures four pixels by four pixels. The frame rate runs at thirty frames per second at the default normal speed. The frame may be turned off to increase the speed of iterations. Time is measured in ticks with one tick equaling one minute in the model.

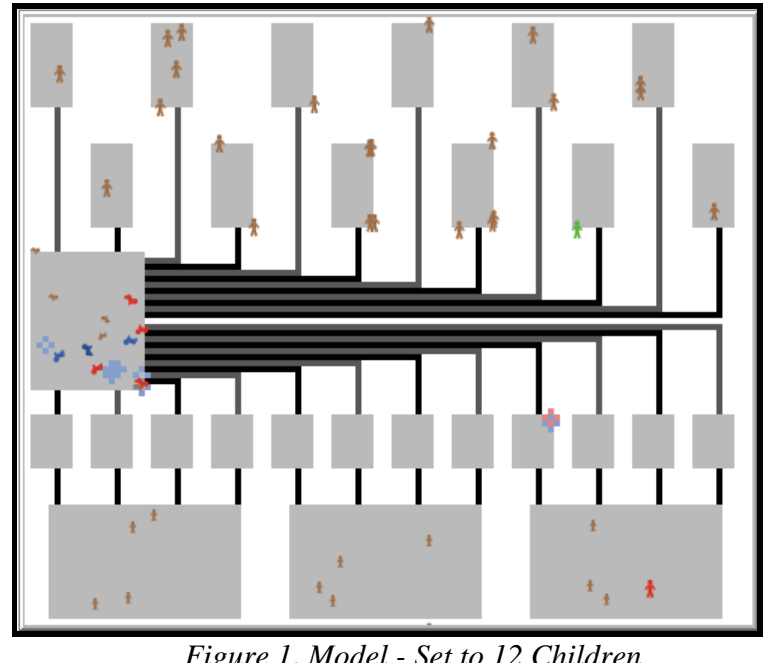

Interactive buttons (Table 2) on the interface include: "Setup!", "Go!", "Go!” (Loop), and "Clear All!".

\begin{tabular}{|c|c|}
\hline Setup! & \multicolumn{1}{|c|}{ Initialize hardcoded and user settings. } \\
\hline Go! & Steps forward one tick (minute). \\
\hline Go! $\quad$ & $\begin{array}{l}\text { Steps forward continuously until } \\
\text { clicked again to stop. }\end{array}$ \\
\hline Clear All! & $\begin{array}{l}\text { Clears the model window, outputs, } \\
\text { monitors, clock, and charts. }\end{array}$ \\
\hline Print Results & $\begin{array}{l}\text { Prints the total count of cases, } \\
\text { symptomatic cases, asymptomatic } \\
\text { cases, and the percentage of } \\
\text { symptomatic and asymptomatic cases. }\end{array}$ \\
\hline Export World & $\begin{array}{l}\text { Exports all data from the model into a } \\
\text { CSV file. This file can be used to } \\
\text { reload the model from its current state } \\
\text { at the time the button was clicked. }\end{array}$ \\
\hline
\end{tabular}


Export View

Exports a PNG of screenshot of the model window (Figure 1).

\section{Table 2. Interactive Buttons}

There are four drop-down menus (Table 3) that allow users to select the starting day, month, year, and a case study selector that presents the potential for additional cases to be quickly selectable for demonstration or analysis in the future. Selecting the case study would automatically adjust variable settings to the suggested setting for a particular case study.

\begin{tabular}{|c|c|c|}
\hline \multicolumn{2}{|c|}{ start-day } & \multirow{2}{*}{ Starting day selectable from 1 to 31 . } \\
\hline 11 & $\nabla$ & \\
\hline \multicolumn{2}{|c|}{ start-month } & \multirow{2}{*}{$\begin{array}{l}\text { Starting month selectable from } \\
\text { January to December. }\end{array}$} \\
\hline Septe & mber $\quad \nabla$ & \\
\hline \multicolumn{2}{|c|}{ start-year } & \multirow{2}{*}{$\begin{array}{l}\text { Starting year selectable from } 2000 \text { to } \\
2018 \text {. }\end{array}$} \\
\hline \multirow[t]{3}{*}{2001} & $\nabla$ & \\
\hline & \multicolumn{2}{|c|}{ casestudy-selection } \\
\hline & Tallahas & e, Florida 2013-2014 \\
\hline \multicolumn{3}{|c|}{$\begin{array}{c}\text { Pre-programmed settings can be automatically applied } \\
\text { using the case-study selector. }\end{array}$} \\
\hline
\end{tabular}

An output window (Figure 4) displays a 24 hour clock formatted "dd $: \mathrm{hh}: \mathrm{mm}$ " that tracks the total number of days that have passed. Below that is the date formatted "Month DD, YYYY". Gregorian calendar rules are followed by the clock. If the month is set to "February" and the day is set to " 30 ", then the clock will jump to "March 1" on the second tick. Leap year is acknowledged and included in the model. The day of the week is correctly selected and displayed below the calendar date. A temperature indicator that shows the daily fluctuation of temperature $+/$ - 10 degrees from the average monthly temperature is displayed on the right-hand side.

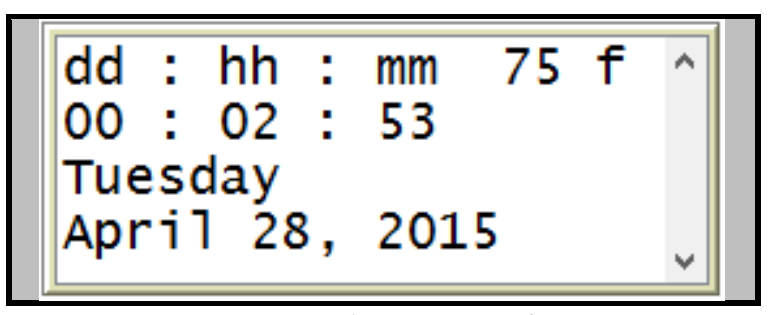

Figure 4. Output Window

Slider bars (Table 3) include: initial-children, pct-initialchildren-vaccinated, pct-initial-parents-vaccinated, pct-initialsiblings-vaccinated, initial-vaccine-effectiveness, antibioticsimmunity, waning-rate-annual, sneezes-per-day, coughs-perday, awareness-strength. Slider bars that range from zero to one or from zero to one-hundred indicate percentages (ie. the slider bar for waning-rate-annual can be set from $0 \%$ to $100 \%$ ). Antibiotics-immunity sets the days that antibiotics will take to stop agent communicability. "Coughs-per-day" and "sneezesper-day" indicate the average per day for a healthy person. Awareness-strength is an agent's assumed ability according to their age to avoid a symptomatic person. This heuristic inference than may be turned on or off at the user's discretion. For this model, the concept that older individuals are more likely to be aware of their surroundings and potentially more likely to avoid symptomatic carriers is used.

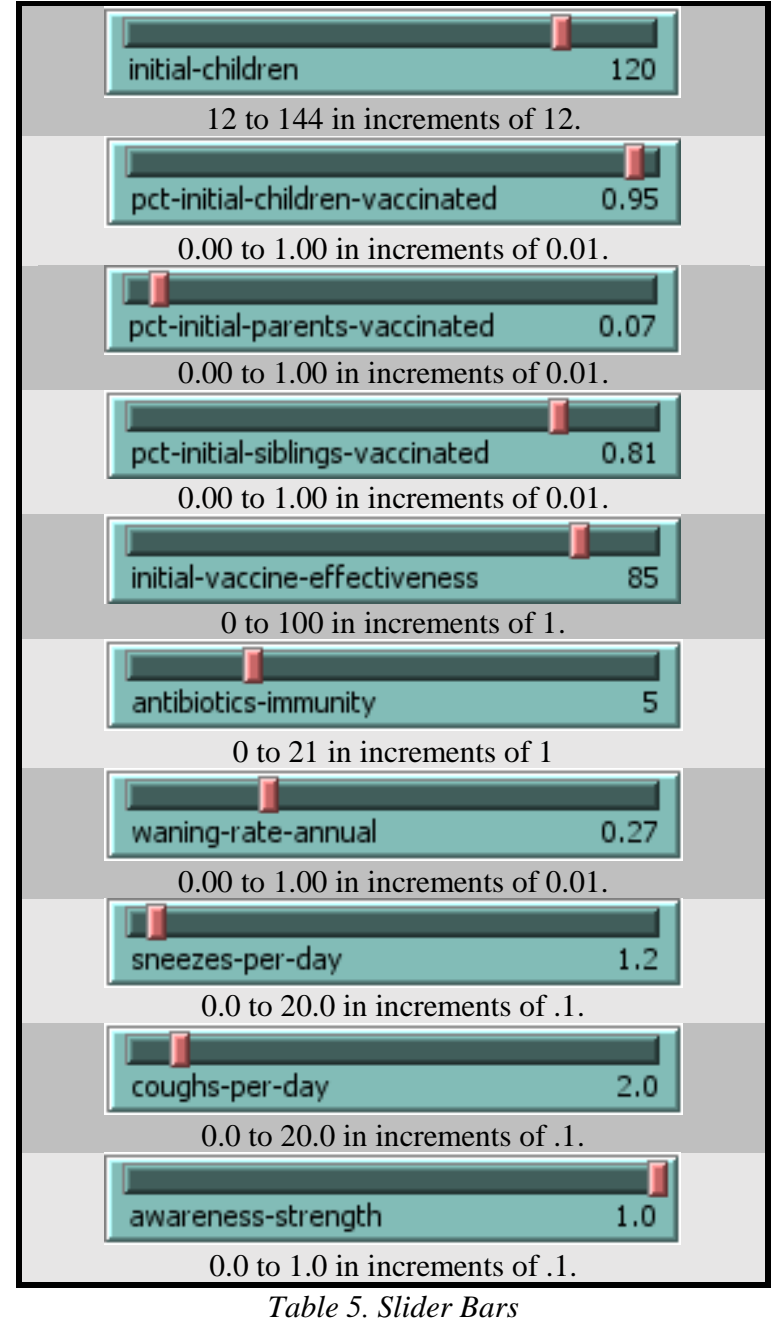

Twelve additional slider bars (Figure 6) indicate the average temperature for any given month and can be adjusted to fit the time period and location of a case study.

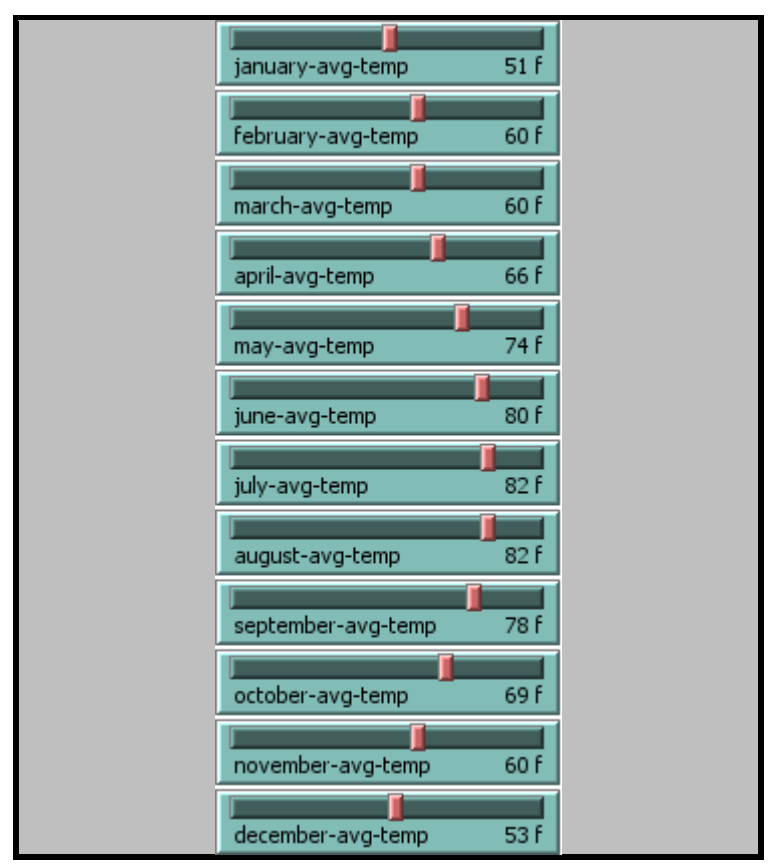

Figure 6. Average Temperature Slider Bars 
There are four Switches (Table 7) that turn on or off asymptomatic carriers, waning immunity, antibiotics, and temperature. If any switch is turned off, then all programming actions regarding that switch will be bypassed in the model. For example, if "antibiotics?" is turned off then agents will not have the opportunity to take antibiotics in order to shorten the disease lifespan and contagious period. These switches are very useful in determining the impact of these confounding variables. Future research could measure these variables by simulating outbreaks with and without these variables turned on and then measuring the difference in outcomes. Aforementioned variables, such as awareness-strength or coughs-per-day may also be made negligible by setting the slider bar to zero.

\begin{tabular}{|l|}
\hline Doff asymptomatic-carriers? \\
\hline Doff waning-immunity? \\
\hline Oon On antibiotics? \\
\hline Doff \\
\hline Don temperature? \\
\hline Off ton \\
\hline
\end{tabular}

Table 7. Switches

A monitor (Figure 8) counts the iterations the model has ran including the iteration currently running.

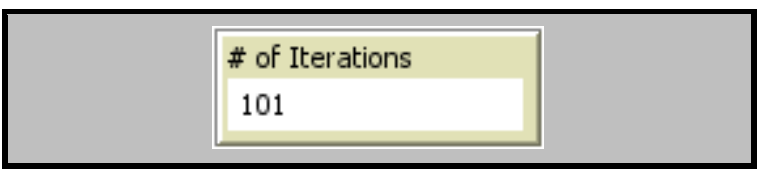

Figure 8. Iterations Monitor

\subsection{Model Infrastructure}

In the model window (Figure 1), the twelve vertical staggered gray rectangles at the top of the window represent workspaces. The workspaces measure eight patches ( 16 meters) horizontal by fifteen patches (30 meters) vertical.

There is a transportation network alternating in color between dark gray and black, to create visually separation, that connects the workspaces to the daycare. While this transportation network is not spatially proportional to a real-world commute, it is methodically suited to the temporal scale. There is an assumption that some daycare enrolled agents will live closer to the daycare while some will live further from the daycare. From left to right, in the bottom row, commute times according to distance and speed are 2, 8, 16, 24, 30, and 38. From left to right, in the top row, commute times in minutes are: $8,13,19,26,34$, and 41. The agents move at a slower rate of speed as they approach corners and buildings. On straightaways, the agents move at their fastest speeds.

The large gray rectangle centered vertically on the left of the window is a daycare classroom. It measures 20 patches (40 meters) horizontal by 24 patches (48 meters) vertical.

There is a transportation network alternating in color between dark gray and black, to create visual separation, that connects the daycare to the houses. While this transportation network is not spatially proportional to scale it is heuristically accurate on a temporal scale. From left to right, commute times in minutes are: $2,2,7,12,18,23,29,34,40,45,51$, and 56 .

The twelve vertical gray rectangles connected to the transportation network below the daycare represent houses. The houses measure eight patches (16 meters) horizontal by ten patches (20 meters) vertical.

The transportation network below the houses has a commute time of 6 minutes from house to school or school to house.

The three horizontal gray rectangles at the bottom of the model represent schools. The schools measure thirty-three patches (66 meters) horizontal by twenty patches (40 meters) vertical.

\subsection{Agents}

All agents are initially brown which indicates that they have never been colonized by Pertussis Bacteria (Figure 9). Agent sizes are intentionally exaggerated on the schematic, although their personal space is only a single patch ( 2 meters by 2 meters). All agents are given an awareness factor that is equal to their age. The awareness factor is multiplied by the awareness-strength that is set by the user. When an agent is challenged by bacteria carried by a symptomatic person, there is an awareness-factor * awareness-strength chance in 100 that they will avoid becoming contaminated. If this heuristic feature is undesired a user can set the awareness strength to zero.

The number of children vaccinated or unvaccinated is dependent on the slider bar (Table 5) selection of the user. Children are assigned ages according to the house they are assigned. The first twelve houses have children ages .5 months to six months in increments of .5 months. The children continuously and proportionally increase in age for each house, with the last agent being .5 month older for every twelve houses. When the initialchildren slider bar for initial-children is set to greater than twelve this pattern continues. For example, if the initial-children slider were set to twenty-four children, the child in the twenty-fourth house would be one-year-old. As the model is running, the agents age at a rate consistent with the Gregorian calendar, including leap year. Each day that passes will age the infant one day and dependent variables such as vaccination effectiveness and awareness-factor are adjusted accordingly.

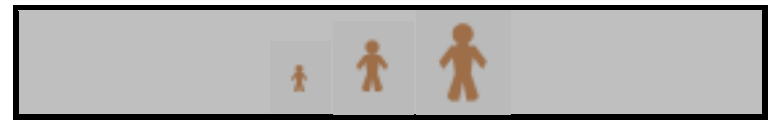

Figure 9. Initial Child, Sibling, and Parent

The ruleset for implementing a vaccination schedule was modeled directly after the Recommended Immunization Schedule for Children and Adolescents Aged 18 Years or Younger (United States 2017) (CDC 2016a). Children receive their vaccinations at two months, four months, six months, fifteen months, and four years of age.

If the waning-immunity switch is turned on, then the vaccine effectiveness will decrease at a constant pace, daily, according to the waning-rate-annual slider bar setting. When initially created, agent's vaccine effectiveness is automatically waned to fit their age and last vaccination according to the vaccine schedule.

The number of parents vaccinated or unvaccinated is dependent on the slider bar (Table 5) selection of the user. The parents last 
vaccination is set by the parent's age minus the age of their child. It is assumed that parents who vaccinate themselves will get a vaccination when their child is born and no additional vaccinations are taken after that. The parent's vaccination effectiveness will wane at the rate indicated on the waning-rateannual slider bar. Parents are all assigned a random age between twenty-three and forty years old. For purposes of pragmatism, it can be assumed that an sixteen-year old sibling with two twentythree-year old parents was adopted or has otherwise entered a non-traditional living arrangement. One parent is a care-giver parent which uses the transportation route to take the child to daycare and then follows the transportation network to their assigned workspace. This parent is assigned to the workspace that is directly above their house. The non-care-giver parent is randomly assigned to a workspace.

The number of siblings vaccinated or unvaccinated is dependent on the slider bar (Table 5) selection of the user. Each sibling's last vaccination is randomly set between four and six years old according to the recommended CDC vaccination schedule referenced above. The sibling's vaccination effectiveness will wane at the rate set on the waning-rate-annual slider bar. The sibling goes to the school that is connected by a transportation network, directly below them and potentially interacts with one of the other three peers from their neighborhood.

\subsection{Pertussis Transmission and Infection}

A symptomatic carrier may transmit bacteria up to a two-patch (4 meter) radius surrounding themselves. The four-meter radius for Bordetella Pertussis bacteria droplet transmission was derived from a controlled study conducted in a hospital (Aintablian, 1998). Transmission of bacteria does not occur across transportation networks. This maintains the representation of a real-world transportation network in which paths traveled from home to daycare would vary and children with their parents would be within the confines of their vehicle. Future developments could include public and other types of potential alternative transportation networks.

Agents within the 4-meter radius are not challenged if they have acquired natural immunity from being previously infected with Pertussis. This setting is supported by a reproduction simulation of an outbreak in Australia, natural immunity was required to last more than fifty years for $90 \%$ of the simulated population to obtain reflective results (Campbell, 2015). This research reflects complete protection from naturally derived immunity, meaning agents cannot acquire Pertussis twice in the same iteration.

Agents within the four-meter radius, when an infected agent sneezes or coughs, are also tested to see if they are already colonized by bacteria. If the agent is already colonized by bacteria, they are not transmitted to twice, the colonized agent continues in the regular progression of the disease phase. The model tests the awareness factor to offer a chance for healthy individuals to avoid infection from a symptomatic carrier. If an agent is not naturally immune, not already colonized, and the awareness test fails then one is added to the "symptomaticchallenge-count".

After that, the model checks to see if the agent is vaccinated. If the agent is not vaccinated, then having failed the previous tests they become symptomatically infected. If the agent is vaccinated, the vaccine effectiveness is tested to determine if the vaccinated individual will become infected as a symptomatic or asymptomatic carrier. If the vaccine fails, the agent becomes a symptomatic carrier. If the vaccine succeeds then the agent becomes colonized as an asymptomatic carrier but not infected as a symptomatic carrier. If asymptomatic carriers are turned off, then an agent cannot become an asymptomatic carrier and nothing happens to the agent when vaccination defense is successful. After a symptomatic infection, the model window is marked by four light red patches surrounding the occurrence of the infection to indicate a symptomatic transmission occurred at that location and one is added to the "cause-symptomaticcounter".

Transmission from an asymptomatic carrier goes through the same testing process prior to a symptomatic or asymptomatic infection. The chance of avoidance of infection due to the awareness-factor is reduced by $25 \%$ due to the agent not being a symptomatic carrier which could be avoided by a person aware of their surroundings. A challenge or cause of infection is accredited to the asymptomatic counter rather than the symptomatic counter. Also, the neighboring patches are set to a light blue color to indicate an asymptomatic cause of infection, rather than a light red color.

\subsection{Disease Lifespan}

Upon infection, asymptomatic and symptomatic carriers are tracked and counted using "track-symptomatic", "symptomaticcounter", "track-asymptomatic", and "asymptomatic-counter" global variables. The infected agents are individually assigned random lengths within specified ranges of disease phases: incubation ( 7 to 14 days), catarrhal (7 to 14 days), paroxysmal (14 to 28 days), and convalescent ( 7 to 21 days). A phase-tracker variable tracks the individual agent's length of time since infection and progresses the agent through the phases as applicable. Symptomatic agents are red and asymptomatic agents are blue. The color of the agent visibly darkens in hue as they progress through the phases.

Agents are not contagious during the incubation phase. Agents are contagious during the catarrhal phase and the paroxysmal phase. During the catarrhal phase there is a chance that once in every seven days a symptomatic agent will take antibiotics and begin the process toward not being contagious and reducing the length of illness. During the catarrhal phase an asymptomatic agent randomly coughs and sneezes each day according to the slider bars (Table 5). In the catarrhal phase of this model, a symptomatic agent sneezes and coughs twice as often as a healthy or asymptomatic agent. The frequency of coughs and sneezes are also affected by temperature. Authors of a study regarding sneezing and coughing noted that "the numbers of sneezes and of nose blowing were lower than expected" (Hansen, 2002) likely due to that "in the winter time, nose blowing is more frequent due to the stimulation of nasal cholinoceptors from cold air (Østberg et al., 1987). This research assumes that the same would be true for sneezing and derives that respiratory coughing is more likely during cold weather.

Due to the increased severity of coughing during the paroxysmal phase there is a once in five days' chance that agents will take antibiotics during the paroxysmal phase. In the paroxysmal phase of this model, symptomatic agents sneeze and cough four times as often as asymptomatic or healthy agents. 
Agents are no longer contagious during the convalescent phase. If an agent takes antibiotics during the catarrhal or paroxysmal phase, then they will become recovered and immune after the number of days set on the slider bar (Table 5) pass. Recovery of agents are tracked. The recovered agent is given natural immunity and turns green to depict the healthy status and natural immunity of the agent in the model window.

\subsection{Agent Movements and Routine}

Admittedly, from a temporal scale, the agents would move very slow if their movement (one meter per minute in buildings) spatially reflected life. The heuristic approach taken in the application of the transportation network is taken for in building agent movements as well. At 0600 all agents "wake up" and begin to move.

Children turn in any random direction and move forward one patch per tick (minute). In this way children's movements are more sporadic and less planned in the model window as might be observed in life. Children can only move within buildings during this time. At 0700 each child goes at the same time as the care-take parent to the transportation network connected to their house. Each child follows the transportation network with the care-taker parent until arriving at the daycare. At the daycare, the child continues to randomly turn 360 degrees and steps forward if a building patch is in front of them. The child potentially interacts with other children during this time. At 1658 each child goes to their transportation network which is connected to the daycare and they wait for their care-taker parent to pick them up. When the parent arrives, the child proceeds on the transportation network back to their house. The child moves around the house in the same manner as described above until 2000, potentially interacting with family members. Each child stops movement at 2000 until 0600 the next morning.

The parents turn in a random direction within a 180-degree window in front of them. This simulates a more directed and purposeful style of movement with less apparent random intent in the model window. The parent can move forward only in buildings unless it is a specified time to take the transportation network. The care-giver parent goes to the transportation network with the child at 0700 . They follow the transportation network and drop off the child. After dropping off the child, the care-taker parent immediately proceeds to their transportation network that connects to their assigned workspace. Then, the care-giver parent proceeds to the workspace. At 0859 after the sibling has gone to school, the non-care-giver parent goes to a random workspace (the parent continues movement within this workspace for the duration of the iteration) that could be connected to any neighborhood, making this parent a potential carrier of the disease across neighborhoods. Each parent moves around the workspace and potentially interacts with other parents. The non-care-giver parent returns home at 1459 to meet the sibling agent and continues movements around the house. The care-giver parent goes to the transportation network to go pick up the child at daycare at 1659. The care-giver parent picks up the child and returns home via the transportation network. The parents move around the house in the same manner until 2100 , potentially interacting with family members, at which time the parents stop movement until 0600 the next morning.
The sibling turns in a random 270-degree window. This reflects the age of the child with less directed movement than the parent, but more directed movement that the child. The sibling moves forward if the space is inside of a building. The sibling is only permitted to move inside of buildings unless on the transportation network at a specified time. At 0800 the sibling goes to the transportation network and then goes to school. The sibling moves around the school and potentially interacts with other siblings. At 1500 hours the sibling goes to the transportation network and returns to their house. The sibling moves around in the same manner until 2100 at which time the sibling stops movement until 0600 the next day.

Parents, children, and siblings stay home on weekends and holidays. The daycare workers move in the same manner as the parents within the daycare during operating hours for the daycare.

\section{RESULTS}

Final results were obtained by running the model through 75 iterations with agent zero being infected on June 1, 2013 and modeling ceasing when there was no longer a possibility of infection or on January 26, 2014. January 26, 2014 is the start of the week following the last reported Pertussis case which fell within the time period of the questionnaire from the Florida daycare case study. After seventy-five iterations, the total number of cases in all iterations comprised 133 daycare workers, 1514 children (0-5years), and 3474 family members. The average per iteration was 2 daycare workers, 20 children, and 46 family members. The average number of children and daycare workers reported as confirmed or probable during the simulation was very similar to the Florida case study while the number of family members infected was $76 \%$ greater than those reported in the case study. The Florida daycare case study had 26 children (students), 2 staff (daycare workers), and 11 family members (Household) who were classified as confirmed or probable via a questionnaire.

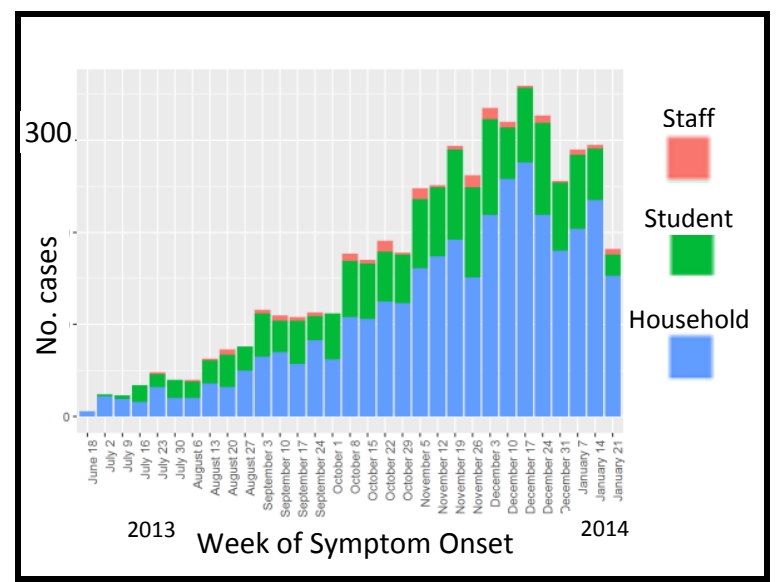

Figure 10. 75 Combined Totals from 75 iterations organized by Week of Symptom Onset 




Figure. Epidemic curve of confirmed and probable pertussis cases during an outbreak in a preschool, by week of symptom onset, Florida, USA, 2013-2014. A total of 26 students (black bars), 2 staff (gray bars), and 11 household/other epidemiologically linked persons (white bars) were involved in this outbreak.

Figure 11. Week of Symptom Onset Epidemic Curve from Florida Case Study

The lifespan of the disease does effectively begin in June and peak in December in the same manner as the Florida case study.

The most interesting result of the model is the conceptualization of potential asymptomatic carrier impact on Pertussis outbreaks. Although, methods such as the questionnaire used to gather information directly from adults affected by the Pertussis outbreak in Florida are useful, the degree of accuracy is limited to the surveyed person's disposition, memory and understanding of medical Pertussis diagnosis as imparted to them by their experiences and the survey or surveyor. The survey demarcates boundaries on what a confirmed or probable Pertussis case is according to medically derived set of criteria. These criteria were used for the model's determination of what a confirmed or probable case would be to the extent of the model's current capabilities and variables. Conditions such as apnea, cough $>2$ weeks, inspiratory whoop, paroxysmal cough, or postussive vomiting are necessary to have a Pertussis case defined as confirmed or probable (Matthias, 2016). Asymptomatic carriers would most likely not exhibit these traits and would fail to get screened by healthcare professionals, have their cases included in surveys, or even be likely to be treated with antibiotics to shorten the duration of their period of being contagious.

Agent based modeling has the potential to offer a unique perspective on an aspect of Pertussis prevalence that has been largely overlooked as a primary factor in the spread of the Pertussis disease until recently, asymptomatic carriers. The combined results from the 75 iterations simulated 5,121 total Pertussis cases which were considered to have met the criteria for being identified as probable or confirmed by the Florida case study questionnaire. The identified confirmed or probable cases accounted for only $25.45 \%$ of the total symptomatic and asymptomatic cases. The asymptomatic and symptomatic cases came to a total of 20,122 across all iterations. 7,404 (36.8\%) of those cases were asymptomatic carriers.

Although, the asymptomatic carriers only accounted for an average of $36.8 \%$ of the total cases throughout the four-month period these agents challenged naïve agents with bacteria almost $4 \mathrm{x}$ as often as symptomatic carriers. This is most likely due to symptomatic carriers having the potential to receive antibiotics which can reduce the duration that they are contagious and capable of transmitting bacteria.
Further calibration on the model is needed to progress to a confirmatory model with the potential to become a predictive model. This first calibration and validation study for using agent based modeling for the fine-scale spatio-temporal analysis of Pertussis as a method for measuring the potential impact of Asymptomatic carriers is conservative when considering that the Pertussis outbreak Florida case study only reported 39 cases. Successes from the model include the demonstration of how "asymptomatic carriers may drive pertussis outbreaks" without detection as remarked by the World Health Organization in August 2015 (WHO 2015). More cases will improve the accuracy of the measurable depiction of asymptomatic carriers as different locations and time periods are incorporated into the model to improve calibration and continue the process of validation.

This is the first agent based model to offer a method for measuring the impact of asymptomatic carriers in Pertussis outbreaks. The possibilities for agent based modeling to contribute to epidemiological studies and other healthcare oriented tasks have only begun to be explored by researchers. Agent based modeling could potentially allow for demonstrations of outbreaks to students entering the healthcare field or in community healthcare campaigns to raise awareness. Customizable agent based models such as this one could potentially make significant contributions to research, community health outreach efforts, and in improving our understanding of Pertussis outbreaks through the measurement of confounding variables and realization of emergent properties such as asymptomatic carriers. 


\section{REFERENCES}

Aintablian, N, Walpita, P, \& Sawyer, M. 1998. Detection of Bordetella pertussis and respiratory synctial virus in air samples from hospital rooms'. Infection Control and Hospital Epidemiology. 19, 12, pp. 918-923. MEDLINE Complete. EBSCOhost. (last accessed 4 May, 2017).

Althouse, B.M. and Scarpino, S.V.. 2015. Asymptomatic transmission and the resurgence of Bordetella pertussis. BMC medicine, 13(1), p.146

Axelrod, Robert. 2001. The Complexity of Cooperation: AgentBased Models of Competition and Collaboration. Princeton University Press, Princeton.

Barclay, L. 2013. Acellular Pertussis Vaccine May Not Prevent Transmission. Medscape Medical News. 2 December.

Campbell, P, McCaw, J, McIntyre, P, \& McVernon, J. 2015. Defining long-term drivers of pertussis resurgence, and optimal vaccine control strategies. Vaccine, 33, pp. 5794-5800. ScienceDirect EBSCOhost. (last accessed 4 May 2017).

CDC. 2015a. Pertussis Cases by Year (1922-2015). https://www.cdc.gov/pertussis/surv-reporting/cases-byyear.html. (last accessed 19 February 2017).

CDC. 2016a. Recommended Immunization Schedule for Children and Adolescents Aged 18 Years or Younger (United States

https://www.cdc.gov/vaccines/schedules/downloads/child/0-

18yrs-child-combined-schedule.pdf (last accessed 4 May 2017).

CDC. 2016b. A Polio-Free U.S. Thanks to Vaccine Efforts. https://www.cdc.gov/features/poliofacts/ (last accessed 19 February 2017).

Domenech de Cellès Matthieu, M. G. Magpantay Felicia, King Aaron A., Rohani Pejman. 2016. The pertussis enigma: reconciling epidemiology, immunology and evolution. Proc. R. Soc. B 2016283 20152309; DOI: 10.1098/rspb.2015.2309. (last accessed 26 February 2017)

Doroshenko A., Qian W., \& Osgood Nathaniel D. 2016. Evaluation of outbreak response immunization in the control of pertussis using agent-based modeling, Peerj, Vol 4, P E2337, p. e2337, Directory of Open Access Journals, EBSCOhost, (last accessed 2 May 2017).

E., N. 1945. "Theory of games and economic behavior (Book Review)." Journal Of Philosophy 42, 550. Book Review Digest Retrospective: 1903-1982 (H.W. Wilson). EBSCOhost (last accessed 1 May 2017)

Finger H, von Koenig CHW. 1996. Bordetella. Baron S, editor. Medical Microbiology. 4th edition. Galveston (TX): University of Texas Medical Branch at Galveston. Chapter 31. https://www.ncbi.nlm.nih.gov/books/NBK7813/ (last accessed 26 February 2017)

Girard, M. P., \& Plotkin, S. A. 2012. HIV vaccine development at the turn of the 21 st century. Current Opinion In HIV And AIDS, 7(1), 4-9. doi:10.1097/COH.0b013e32834ddc96
Guimarães, T. M. R., Alves, J. G. B., Tavares, M. M. F. 2012. Impact of immunization measures by the Family Health Program on infant mortality from preventable diseases in Olinda, Pernambuco State, Brazil. Cadernos de Saúde Pública 25 (40 Rio de Janeiro: Escola Nacional de Saúde Pública, 868-876.

Hansen, Bjarne, Mygind, Niels. 2002. How often do normal persons sneeze and blow the nose?*. Rhinology. 40. 10-12.

Just, W., Callender, H., LaMar, M. D., \& Toporikova, N. 2015. Chapter 8: Transmission of Infectious Diseases. Data, Models, and Simulations. Algebraic and Discrete Mathematical Methods for Modern Biology, 193-215. doi:10.1016/B978-0-12-8012130.00008-3

Matthias, James, Pritchard, P. Scott, Martin, Stacey W., Dusek, Cristina, Cathey, Erika, D’Alessio, Rebecca, Kirsch, Majorie. 2016. Sustained Transmission of Pertussis in Vaccinated, 1-5Year-Old Children in a Preschool, Florida, USA. Medscape Education.

Morgenstern, Oskar. 1958, 'Von Neumann, John', Economic Journal, 68, pp. 170-174, Humanities Source, EBSCOhost, viewed 30 April 2017.

Østberg B, Winther B, Mygind N. 1987. Cold air-induced rhinorrhea and high-dose ipratropium bromide. Arch Otolaryngol. 113:160-162.

Pearson, K. 1930. The Life, Letters and Labors of Francis Galton. Cambridge University Press.

Plotkin, S. A. 2014. The pertussis problem. Clinical Infectious Diseases. 58(6), 830-833.

Schelling, Thomas C. 1968. "Game Theory and the Study of Ethical Systems.". The Journal of Conflict Resolution. vol. 12, no. 1, pp. 34-44. www.jstor.org/stable/172812.

Sanstead, E, Kenyon, C, Rowley, S, Enns, E, Miller, C, Ehresmann, K, \& Kulasingam, S. 2015. Understanding trends in pertussis incidence: An agent-based model approach. American Journal Of Public Health. 105, 9, p. e42-e47. Scopus®. EBSCOhost. (last accessed 30 April 2017).

Warfel, J, Beren, J, Kelly, V, Lee, G, \& Merkel, T. 2012. Nonhuman Primate Model of Pertussis, Infection and Immunity, 80(4): 1530-1536.

Warfel, J., Zimmerman, L., \& Merkel, T. 2013. Acellular pertussis vaccines protect against disease but fail to prevent infection and transmission in a nonhuman primate model. Proceedings Of The National Academy Of Sciences Of The United States Of America, 111(2): 787-792.

Warfel, J., Papin, J., Wolf, R., Zimmerman, L., \& Merkel, T. 2014. Maternal and Neonatal Vaccination Protects Newborn Baboons From Pertussis Infection. Journal Of Infectious Diseases, 210(4): 604-610.

Weber C., Boursaux-Eude C., Coralie G., Caro V., Guiso N. 2001. Polymorphism of Bordetella pertussis Isolates Circulating for the Last 10 Years in France, Where a Single Effective WholeCell Vaccine Has Been Used for More than 30 Years. Journal of Clinical Microbiology, 39(12), 4396-4403. 
http://doi.org/10.1128/JCM.39.12.4396-4403.2001

accessed 26 February 2017)

WHO. 2015. Pertussis Vaccines: WHO position paper. Weekly Epidemiological Record. 28 August. 90(35), 433-460.

WHO. 2014. INFORMATION SHEET OBSERVED RATE OF VACCINE REACTIONS DIPHTHERIA, PERTUSSIS, TETANUS $\quad$ VACCINES. http://www.who.int/vaccine_safety/initiative/tools/DTP_vaccin e_rates_information_sheet.pdf?ua=1. (last accessed 26 February 2017). 